\title{
A Disruptive Treatment to Prevent Amputation and Enhance Limb Salvage in an Elderly Patient
}

\author{
Sarah Cristina Castillo Benitez, Rosa Alduey Duran, Pilar Matos-Ruiz, Madalene C. Y. Heng*, \\ Thomas L. Vander Laan
}

Department of Medicine/Dermatology, UCLA School of Medicine, Los Angeles, USA

Email: *madaleneheng@aol.com

How to cite this paper: Castillo Benitez, S.C., Alduey Duran, R., Matos-Ruiz, P., Heng, M.C.Y. and Vander Laan, T.L. (2019) A Disruptive Treatment to Prevent Amputation and Enhance Limb Salvage in an Elderly Patient. Journal of Cosmetics, Dermatological Sciences and Applications, 9, 129-154.

https://doi.org/10.4236/jcdsa.2019.92012

Received: March 10, 2019

Accepted: June 2, 2019

Published: June 5, 2019

Copyright $\odot 2019$ by author(s) and Scientific Research Publishing Inc. This work is licensed under the Creative Commons Attribution International License (CC BY 4.0).

http://creativecommons.org/licenses/by/4.0/

\begin{abstract}
Background: Chronic wounds are a growing problem internationally, termed a silent epidemic. To combat this epidemic, it is not sufficient to rely on traditional wound care treatments alone, but to look to innovative and alternative therapies. The indispensable role of oxygen in wound healing is well-discussed in the literature, and in the past two decades the topical application of oxygen has shown promising results in the healing of chronic wounds. However, the toxic effects of oxygen are usually not appreciated and can often lead to wound necrosis and gangrene in wounds with compromised blood supply. While wounds with adequate blood supply contain free radical quenchers (catalase, superoxide dismutase and reduced glutathione) which neutralize the free radicals (reactive oxygen species) released as a by-product of the Krebs cycle, wounds with deficient blood supply are deficient in free radical quenchers and are further damaged by exposure to oxygen as a result of reperfusion injury/oxygen toxicity. Topical hyperbaric oxygen (THOT ${ }^{\odot}$ ) uses low oxygen tensions in the hyperbaric range to stimulate angiogenesis, while preventing excessive oxygen toxicity. The result is the induction of marked angiogenesis, with increasing capacity for quenching reactive oxygen species, resulting in wound healing of ischemic wounds. Case Presentation: This case report describes a 95-year-old Caucasian female who presented with a stage IV chronic necrotic ulcer on her lower left leg. Surgical pathology results revealed calcifying vasculopathy, which is thought to be responsible for deficient blood supply to the leg, leading to a necrotic, chronic leg ulcer of her left leg. After traditional wound therapies were unsuccessful, she was considered for leg amputation. The introduction of adjunctive treatment with topical hyperbaric oxygen therapy saw complete healing of the wound within 15 weeks. The wound remained closed, without the presence of scar tissue, with no signs of wound breakdown at three-month and six-month follow-ups. Conclusions: Chronic hypoxic wounds with vascular insufficiency are con-
\end{abstract}


sidered "unlikely to heal" and tend to lead to limb amputation. The use of $\mathrm{THOT}^{\oplus}$ technology, with low hyperbaric oxygen tensions to neutralize free radicals released by the Krebs cycle when oxygen contacts the wound, prevents oxygen toxicity and results in angiogenesis necessary for wound healing. In this way, $\mathrm{THOT}^{\oplus}$ treatment was able to convert the hypoxic "unlikely to heal" wound considered for limb amputation into one which healed, with limb salvage. The results of this case report demonstrate the potential for complete healing of chronic hypoxic wounds even in complex cases with multiple confounding factors preventing wound healing, using a cost-effective treatment that is easily accessible to patients.

\section{Keywords}

Limb Salvage, Necrotic Leg Ulcers, Topical Hyperbaric Oxygen,

Angiogenesis, Hypoxic Wounds, $\mathrm{TcPO}_{2}$

\section{Introduction}

Chronic wounds, defined as wounds that fail to heal, are placing an increased burden on a health care system already encumbered by increased patient morbidity, an aging population, and rising health care costs [1] [2]. Often overlooked as a comorbid condition, chronic wounds have been described as a "silent epidemic" [3]. A retrospective analysis of Medicare patients in the USA revealed that nearly 8.2 million (15\%) were diagnosed with at least one wound or wound-related infection in 2014. Medicare spending for wound care was also analyzed, revealing an estimated a cost of $\$ 6.4$ billion (USD) for chronic ulcer care that year and a total mid-range estimate of $\$ 31.7$ billion for all wound care expenditures [4]. Broadening the scope, when looking at all USA patients, chronic wounds affect approximately 6.5 million people [3]. An estimated $3 \%$ of the total health care expenses in developed countries is attributed to chronic wound care [2].

The antidote for the chronic wound epidemic involves the identification of innovative yet simple, accessible, and cost-effective interventions. The benefits of oxygen and its key role in wound healing are discussed extensively in the literature, and this evidence points to the simple solution of oxygen therapy as an adjunct treatment for wounds. Oxygen is required for tissue repair and regeneration, necessary in nearly every phase of the wound healing process. Not only is it a component of cellular metabolism, yielding energy for use by the cells in the repair process, but it is also essential for the synthesis of protein and the production of blood vessels and collagen-vital materials in wound repair and quality healing [5] [6]. Oxygen must be present for collagen fibers to cross-link, which increases the tensile strength of tissue [7].

Chronic wounds tend to be hypoxic, or lacking oxygen within the wound tissue [8] [9] [10]. Prolonged hypoxia reduces the proliferation of endothelial cells 
and fibroblasts. These connective tissue cells hold a critical function in various biological processes essential for wound healing. Fibroblasts are involved in the process of collagen deposition, help form granulation tissue whose presence is the hallmark of an established healing response, and produce the glycoprotein Vascular Endothelial Growth Factor (VEGF) [11]. VEGF has been discovered to play a role in collagen deposition and epithelialization, as well as the stimulation of multiple components of the angiogenic cascade. Angiogenesis, or the formation of new blood vessels, restores blood supply and delivers crucial nutrients and oxygen to the surrounding tissue for wound healing.

Besides hypoxia's deleterious effect on fibroblast proliferation and sustained angiogenesis, it also directly inhibits antimicrobial activity, compromising the tissues' ability to fight infection [12]. Oxygen has a microbial growth inhibitory effect (creating a poor environment for anaerobic bacteria to thrive) and acts in neutrophil activation, leading to the phagocytosis of harmful microbes. Hence chronic wounds, suffering from hypoxia, are more prone to infection and lack the necessary energy, vascularization, and collagen production required for effective tissue repair.

\section{Hyperbaric Oxygen Delivered as Topical Hyperbaric Oxygen Therapy: How It Works}

Oxygen's indispensable role in tissue repair has precipitated investigation of oxygen as a wound care intervention. Hyperbaric oxygen therapy is widely known, but the past two decades have seen the rise of an innovative form of oxygen therapy involving the topical application of oxygen to the open wound [13].

Based on transcutaneous oxygen partial pressure measurements $\left(\mathrm{TcPO}_{2}\right)$, hypoxic wounds $\left(\mathrm{TcPO}_{2} 0-30 \mathrm{~mm} \mathrm{Hg}\right)$ are identified by the presence of yellow necrotic slough $\left(\mathrm{TcPO}_{2} 13-30 \mathrm{~mm} \mathrm{Hg}\right)$ or black gangrene $\left(\mathrm{TcPO}_{2} 0-13 \mathrm{~mm}\right.$ $\mathrm{Hg}$ ). Necrotic wounds with $\mathrm{TcPO}_{2}$ between $0-30 \mathrm{~mm} \mathrm{Hg}$ are considered "unlikely to heal" [14], resulting in high rates of surgical interventions, including amputation. These wounds are recognized clinically by recurrent formation of necrotic tissue at the air/wound interface. The necrotic tissue reforms when oxygen contacts the wound surface due to reperfusion injury-i.e. when hypoxic tissue, which lacks the ability to neutralize oxygen free radicals/reactive oxygen species because of deficient blood supply, is reperfused by oxygen from the air.

Unopposed oxygen free radicals or reactive oxygen species, a normal byproduct of oxidative phosphorylation (Krebs cycle) form the basis of reperfusion injury [15] [16] [17]. In non-hypoxic wounds with adequate blood supply, these free radicals are quenched by the free radical quenchers (superoxide dismutase, catalases and reduced glutathione) present within blood vessels; consequently, no necrotic tissue forms when oxygen contacts the wound surface. However, in hypoxic wounds lacking in blood supply, the presence of unopposed free radicals results in further endothelial cell destruction and tissue necrosis, with worsening of these non-healing wounds. Using oxygen pressures low enough to avoid oxygen toxicity as well as high enough with properties to "mop up" oxygen free 
radicals is the basis for the induction of angiogenesis in necrotic wounds. Heng et al. have identified a range of pressures (1.004 - 1.013 atmospheres) with Topical Hyperbaric Oxygen Therapy (THOT ${ }^{\circledR}$ ) which have been observed to induce angiogenesis in necrotic wounds [18].

In this case report, we report complete healing of a hypoxic, non-healing leg wound in 15 weeks with $\mathrm{THOT}^{\circ}$. The patient had been scheduled for amputation. Our hope is that it will prompt further clinical studies and case reports, as well as focus the spotlight further on a simple, yet effective, wound care therapy which should be considered by health care professionals when prescribing treatment regimens.

\section{Case Presentation}

A 95-year-old Caucasian female scheduled for amputation presented with a stage IV non-healing necrotic leg wound. Per the medical records, the patient could not recall a specific injury or event that precipitated the wound. Her past medical history included: osteoarthritis, bradyarrhythmia with pacemaker placement, gastroesophageal reflux disease, osteoporosis, hyperlipidemia, hypertension, urinary incontinence, hysterectomy, and polymyalgia rheumatica.

\subsection{Patient Evaluation and Treatment Prior to the Initiation of THOT ${ }^{\circledR}$}

The patient had been treated through a local wound care center, where her treatment included weekly sharp debridement and daily dressing changes with application of Santyl and gentamicin to the wound bed. Topical hydrocortisone and Silvadene were also implemented at a later stage. Despite these efforts, the wound continued to deteriorate, and the probability of the wound healing was documented as "quite small" by one of the wound care center physicians and the patient was discharged due to an assessment of "inability to heal". The facility recommended that the patient be evaluated for a below-the-knee amputation. The patient's family referred the patient to a surgeon for alternative treatment options due to poor healing with the current treatment regimen in order to prevent amputation.

When first evaluated by the surgeon, the patient's left lateral leg wound had been present for several months. On initial assessment, the surgeon described the wound as measuring $6 \mathrm{~cm}$ in diameter, with necrotic eschar over the wound base (Figure 1). The wound (Figure 1) was very tender to palpation. The surgeon's opinion was that there was a high probability that the wound would not heal due to necrotic tissue, infection, and the patient's age.

A surgical excision was performed, down to the muscular fascia, for removal of the necrotic tissue. The operative report detailed that the necrotic tissue in the ulcer involved the subcutaneous fat and extended to the muscular fascia, although the fascia itself and muscle were viable. The patient's wound after surgical debridement is shown in Figure 2. The patient remained stable throughout the procedure, recovered well, and was discharged home two days later. 


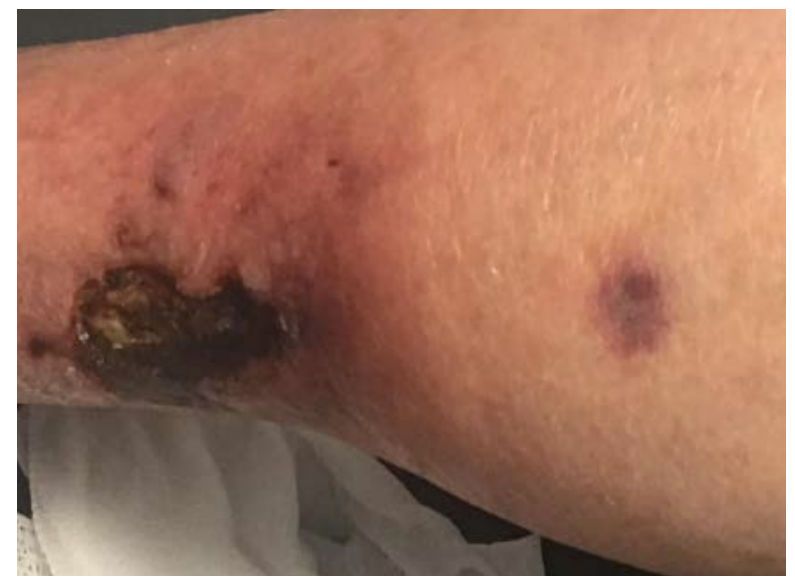

Figure 1. May 2017. Wound appearance on initial evaluation by surgeon. Patient's left lateral leg wound has been present for several months. Note necrotic tissue and swollen area of infection and inflammation.

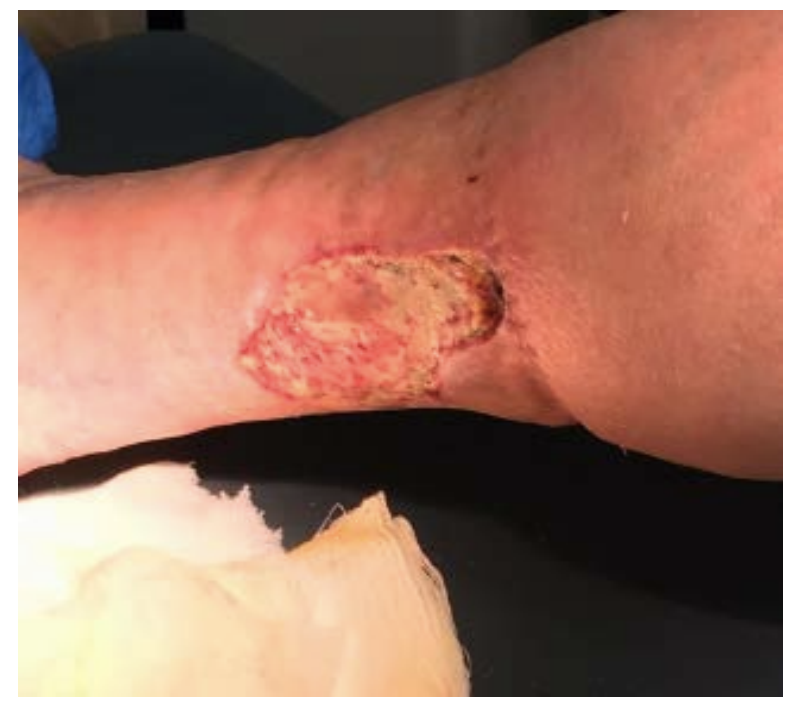

Figure 2. June 1, 2017. $8 \times 5 \mathrm{~cm}$ wound post-surgery. Patient was placed under general anesthesia, regional block, and a nerve block. The ulcer was excised to the muscular fascia. Operative report noted that the necrotic tissue in the ulcer involved the subcutaneous fat and extended to the fascia. Although most of the fascia and muscle were viable, the surgeon was not able to debride all necrotic tissue overlying the muscle e.g. proximal area of wound (upper right in photo) was not debrided. The leg was still infected/cellulitic and swollen.

The surgical pathology report listed a diagnosis of calcifying vasculopathy with histologic findings consistent with calciphylaxis: "Histologic sections demonstrate partially ulcerated skin and subcutaneous tissue with granulation tissue and numerous small to medium arteries exhibiting occlusive endoluminal calcifications." There was no evidence of vasculitis in the biopsy. Cultures obtained at the time of surgery were positive for two strains of Pseudomonas aeruginosa (few) and the gram stain was positive for white blood cells (rare) and gram-negative bacilli (few). Susceptibility results showed no evidence of drug resistance among the $P$. aeruginosa strains identified in the wound. 


\subsection{Prescribed Plan of Care, Status Post-Surgical Excision of Necrotic Wound Tissue}

To enhance the patient's healing post-surgery, the surgeon prescribed Topical Hyperbaric Oxygen Therapy (THOT ${ }^{\circledast}$ ), to be delivered via the Numobag ${ }^{\circledast}$ Kit following surgical debridement The prescription was initially written for 12 Numobag ${ }^{\oplus}$ Kits (a 3-week supply), with 4 refills. In addition to $\mathrm{THOT}^{\oplus}$, the surgeon's plan of care recommendations included:

- Gentamycin ointment to wound bed

- Evaluation of calcium/parathyroid hormone metabolism (calciphylaxis diagnosis)

- Sodium thiosulfate (calciphylaxis diagnosis)

\subsection{Topical Hyperbaric 0xygen Therapy $\left(\right.$ THOT $\left.^{\circledR}\right)$ [18]}

Topical hyperbaric oxygen therapy with oxygen pressures delivered at the therapeutic range $\left(\mathrm{THOT}^{\circ}\right)$ is delivered via a patented device known as the Numobag $^{\oplus}$. Oxygen is administered via an $84 " \times 48$ " pleated polyethylene bag. The open end is taped around the chest at the level of the nipple, allowing multiple ulcers to be simultaneously treated. Using pressures validated by instruments specially designed for measuring low pressures (Sandia, National Labs, Albuquerque, New Mexico), intrabag pressures were maintained within a narrow range (1.004 to 1.013 atmospheres) at all times, as well as ensuring a $15 \mathrm{~L} / \mathrm{min}$ flow rate. Per Numobag ${ }^{\oplus}$ protocol, the patient received THOT $^{\oplus}$ for 4 hours a day, 4 consecutive days per week. In between, the ulcers received regular dressing changes. All treatment was clinically managed in the patient's home. A home-care nurse was trained to administer $\mathrm{THOT}^{\oplus}$ following the Numobag ${ }^{\oplus} \mathrm{Kit}$ protocol. Figure 3 shows the wound just prior to the first THOT $^{\varpi}$ session.

\subsection{Adjunctive Wound Care Interventions}

Strict infection control measures were implemented, including the use of sterile dressing materials and daily linen changes. The wound was complicated by a significant amount of purulent exudate, as evident in Figure 4(a), with decreased exudate following the first treatment with $\left(\mathrm{THOT}^{\circledR}\right)$ (Figure 4(b)).

As reported in the culture obtained during the initial surgical procedure, the wound was found to be colonized with Pseudomonas aeruginosa (Figure 5). In addition to $\mathrm{THOT}^{\oplus}$ treatments four times per week, these complications were addressed with a 4-week course of oral ciprofloxacin and daily dressing changes performed by the home nurse with application of gentamycin ointment to the wound bed. As the wound began to demonstrate signs of healing, Carrasyn hydrogel was added to the patient's wound care regimen to maintain an adequate moisture balance in the wound.

\subsection{Healing of Leg Ulcer with THOT ${ }^{\circledR}$}

During weeks 1 - 5 of $\mathrm{THOT}^{\oplus}$ treatment (Figures 6-10), necrotic tissue was observed to form after debridement, albeit in decreasing amounts from $70 \%$ of 
wound surface (Figure 6), to $15 \%$ of wound surface (Figure 10). This is associated with increasing neovascularization (Figure 10). Epithelialization was observed at week 5 of $\mathrm{THOT}^{\oplus}$ treatment (Figure 10). The above improvement was associated with decreasing purulent exudate (Figures 6-10). At week 6, no necrotic tissue was observed following surgical debridement (Figure 11), indicating that the increased number of new blood vessels generated by the previous 5 weeks of therapy provided sufficient free radical quenchers to prevent further reperfusion injury. This is the first indication that this wound had the potential to heal. At weeks 7 (Figure 12), neovascularization was even more abundant with absence of formation of new necrotic tissue. Re-epithelialization was readily visible, resulting in decreased ulcer size (Figure 12). The wound continued to re-epithelialize over the next 6 weeks (Figures 13-20), with no dermis visible after 14 weeks of treatment (Figure 20). Over the subsequent 6 weeks (Figures 21-27), full thickness epidermis was formed, with maturation of the stratum corneum, and remodeling of the wound vasculature (Figures 24-27).

\subsection{Wound Size over Time}

Weekly measurements of wound size over time show improvements in ulcer size, as documented in Figure 28.

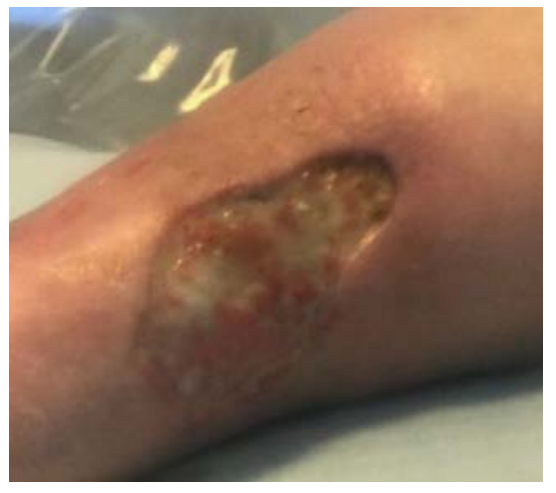

Figure 3. June 27, 2017, Week 1. Wound appearance prior to first topical hyperbaric oxygen treatment. Note rapid formation of necrotic tissue following debridement.

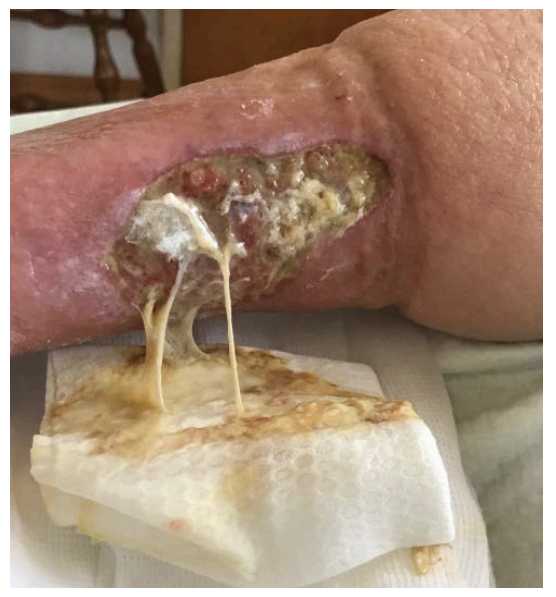

(a) 


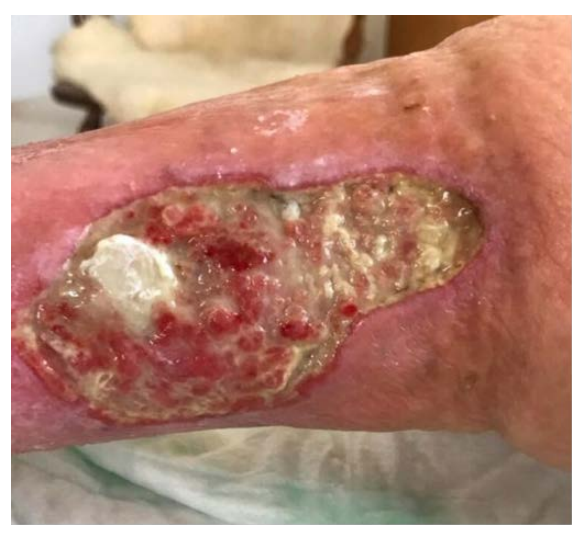

(b)

Figure 4. July 1, 2017, Week 1: (a) Wound before THOT $^{\varpi}$ treatment. (b) Post first THOT $^{\circledast}$ treatment. Note the reduced amount of exudate and beginning of healing after the first $\mathrm{THOT}^{\circledast}$ treatment.

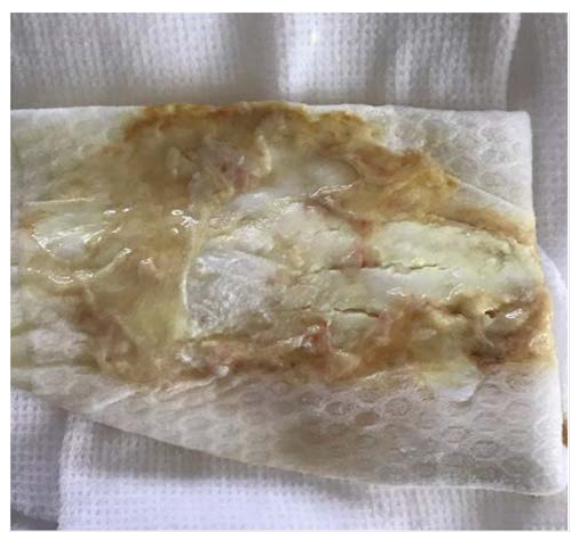

Figure 5. July 1, 2017, Week 1. Dressing change. The wound was compromised by a significant amount of purulent exudate. The wound was also colonized with Pseudomonas aeruginosa which is a common organism cultured from necrotic wounds.

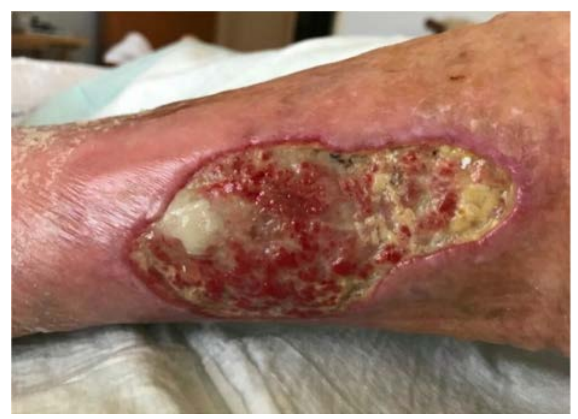

Figure 6. July 4, 2017, Week 1. Post THOT ${ }^{\circledR}$ treatment. Wound was improving, but still infected and necrotic. The surgeon was not able to debride the wound completely. Increased granulation tissue was visible. Adherent yellow necrotic slough was observed, covering $<70 \%$ of wound. Decreased peripheral edema was observed surrounding the wound, now non-pitting. Although new blood vessels had formed in the wound as a result of $\mathrm{THOT}^{\oplus}$, it had only been three days since THOT $^{\circledast}$ was instituted, and the neovascularization was not adequate to provide sufficient free radical quenchers to neutralize all the free radicals released from exposure of the wound to oxygen. The presence of necrotic tissue, produced by reperfusion injury, reflects this deficiency. 


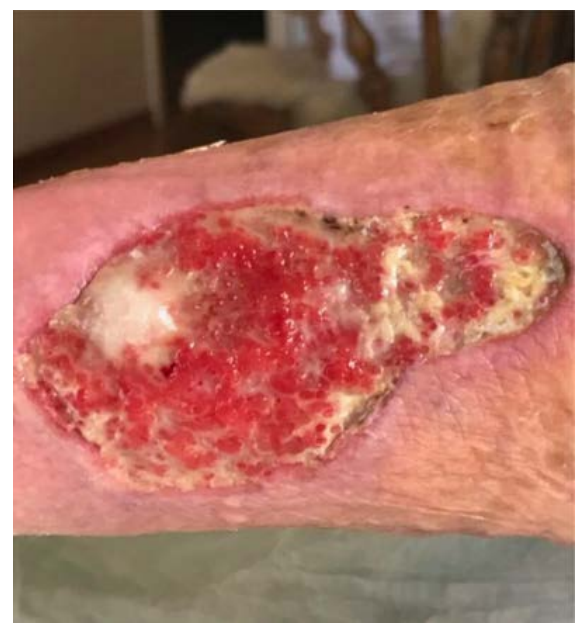

(a)

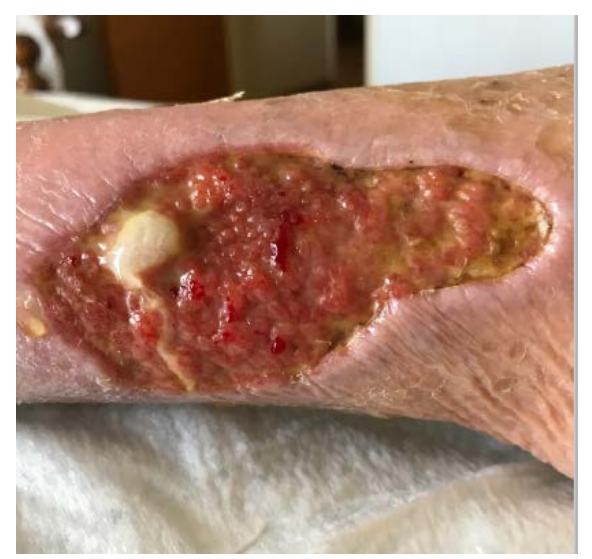

(b)

Figure 7. July 12, 2017, Week 3. (a) Pre-THOT ${ }^{\circledast}$ treatment. (b) Wound now measured $8 \times$ $4.5 \mathrm{~cm}$. Wound edges were less swollen. Increased granulation tissue was observed. Necrotic slough covers $<50 \%$ of wound. Moderate purulent tan/yellow drainage was observed. Decreased peripheral tissue induration was noted (extending $<2 \mathrm{~cm}$ around wound).

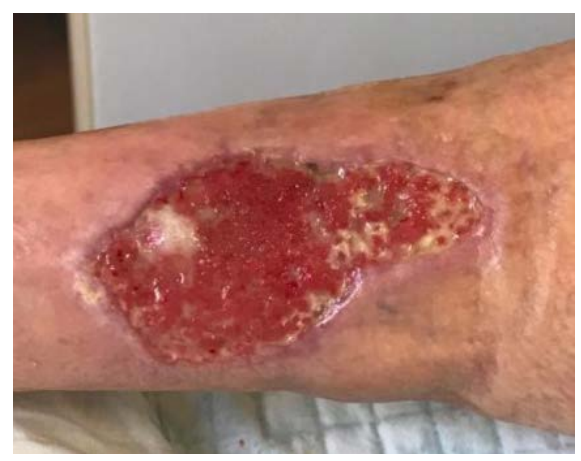

Figure 8. July 18, 2017, Week 4. Pre-THOT ${ }^{\circledR}$ treatment. Epithelialization was observed at wound edges as wound continued to decrease in size (now $7.75 \times 4.5 \mathrm{~cm}$ ). Loosely adherent slough was observed to cover $<25 \%$ of wound, with increased new bright red granulation tissue. Moderate amount of exudate was observed but changed from purulent to serosanguinous. Non-pitting edema to surrounding tissues was present but affected a smaller area around the ulcer. 


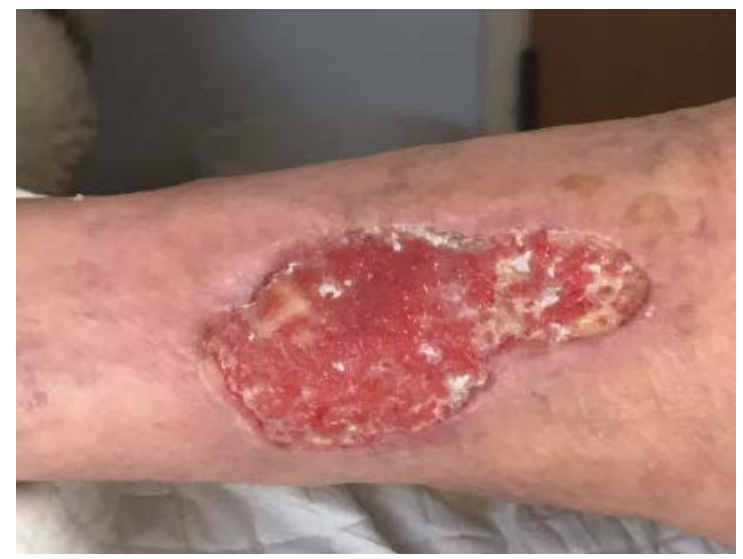

(a)

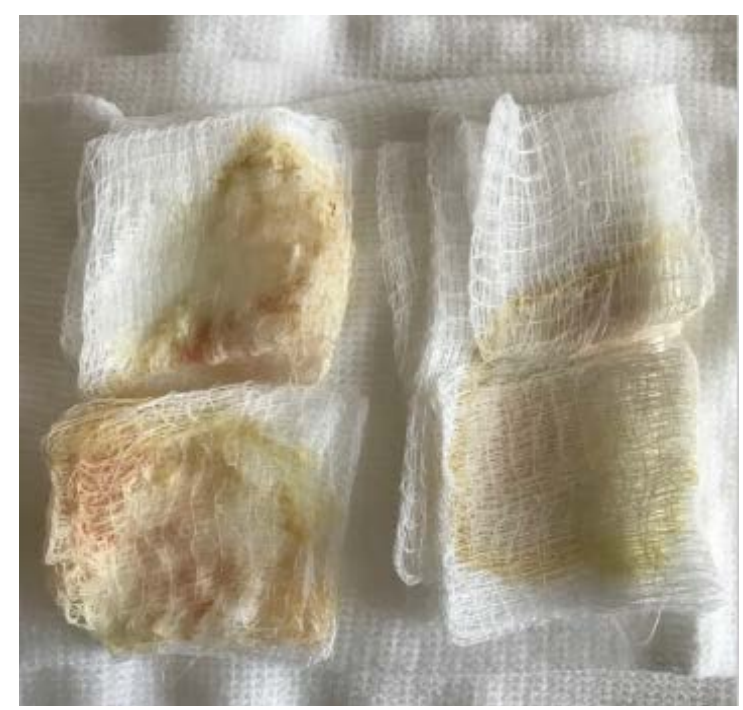

(b)

Figure 9. July 23, 2017, Week 4. (a) After 4 weeks of THOT $^{\varpi}$, wound measured $7.5 \times 4.25$ $\mathrm{cm}$. Peripheral edema continued to decrease (non-pitting). Extensive granulation tissue formation was evident, with yellow slough covering $20 \%$ of the wound surface. (b) Moderate amount of purulent exudate mixed with scant sanguineous drainage.

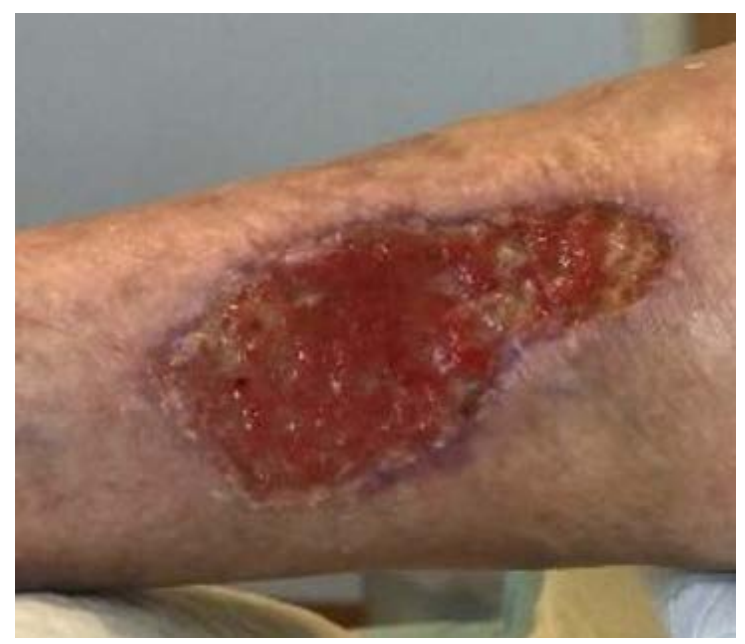

(a) 


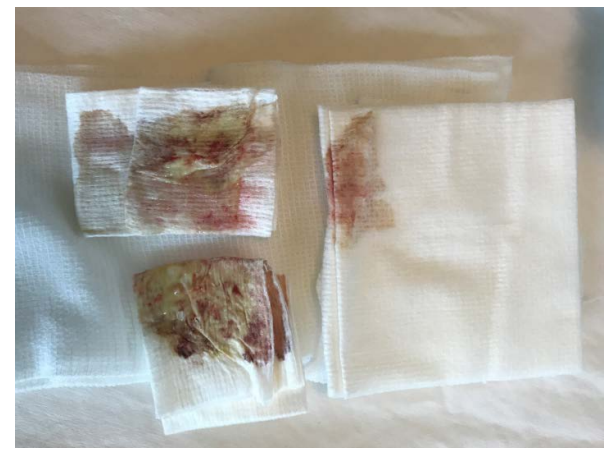

(b)

Figure 10. July 31, 2017, Week 5. (a) Wound measured $6.5 \times 4 \mathrm{~cm}$. Yellow necrotic tissue covered $<15 \%$ of wound bed; mild non-pitting edema was observed in surrounding tissue, and new epithelial tissue was noted all around, with epithelial tissue attached to wound base. (b) Exudate was serosanguinous, not purulent, and had decreased from moderate to just a small amount.

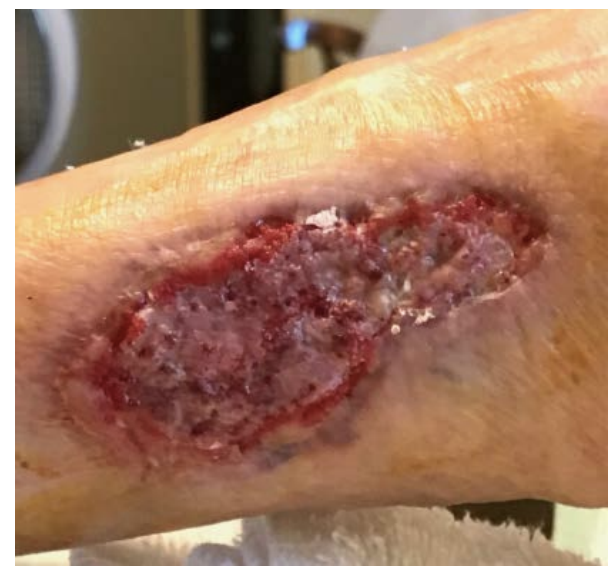

(a)

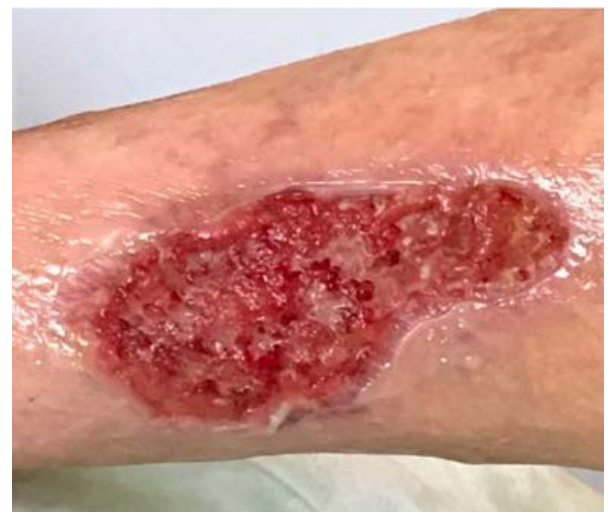

(b)

Figure 11. August 4, 2017, Week 6. (a) Wound post surgical debridement but before THOT $^{\varpi}$ treatment. (b) Wound post-surgical debridement and after THOT $^{\varpi}$ treatment. Note absence of necrotic tissue and accelerated angiogenesis after surgical debridement and same-day THOT $^{\oplus}$ treatment. This indicates that the increased neovascularization generated by 4 weeks of THOT $^{\circledast}$ provided sufficient free radical quenchers to prevent further reperfusion injury when the underlying tissues were exposed to oxygen. This pivotal moment is the first indication that this wound had the potential to heal. 


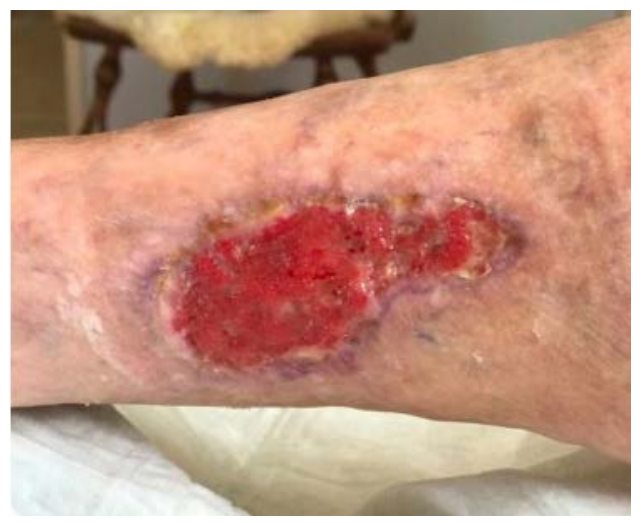

Figure 12. August 8, 2017, Week 7. Wound measured $6.5 \times 3.75 \mathrm{~cm}$. Absence of necrotic slough suggests that the number of new blood vessels formed contained sufficient free radical quenchers to prevent reperfusion injury when wound was exposed to the air or oxygen. There was no edema in surrounding tissue. Instead, there was beefy, red granulation tissue filling $>90 \%$ of wound with evidence of re-epithelialization. Wound has changed significantly. Note the presence of new granulation tissue containing abundant new blood vessels. The purple areas around the edges of the wound represent post-inflammatory hyperpigmentation in the newly formed epidermis. There was significant re-epithelialization associated with attachment of the vascular dermis to the epidermis, which allowed for diffusion of the oxygen from the dermal blood vessels to the epidermis to promote epidermal proliferation and re-epithelialization.

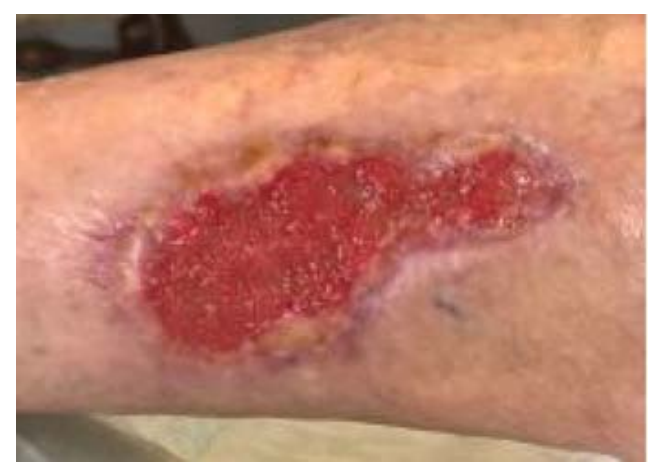

(a)

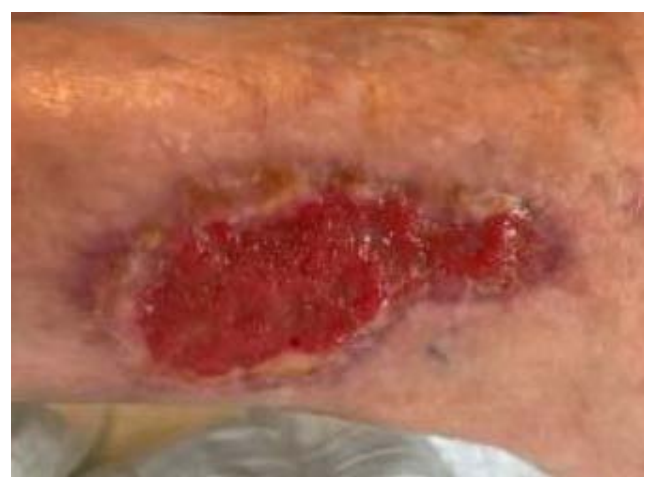

(b)

Figure 13. August 15, 2017, Week 8. (a) Pre-THOT ${ }^{\varpi}$ treatment. (b) Post-THOT ${ }^{\circledast}$ treatment. Wound measured $6.25 \times 3.5 \mathrm{~cm}$. Note the presence of good granulation tissue and re-epithelialization. The major change is the increased amount of oxy-Hb within the blood vessels following THOT $^{\circledast}$ treatment. 


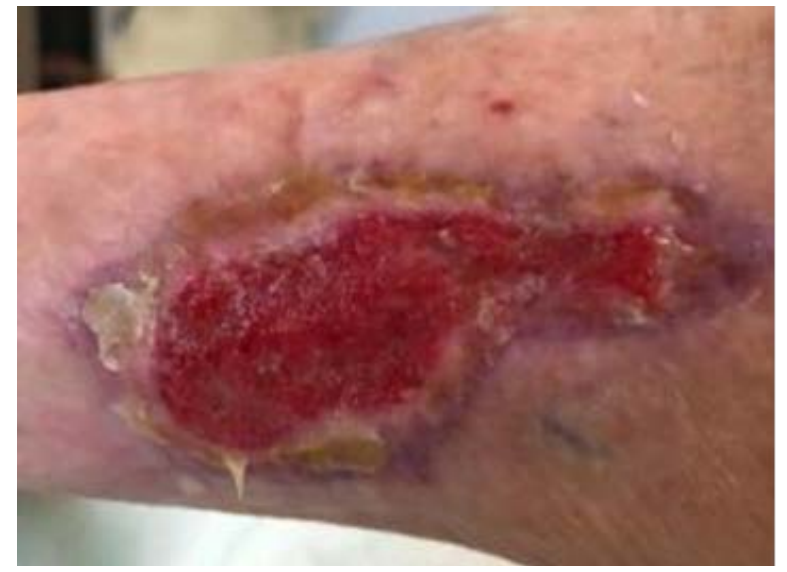

(a)

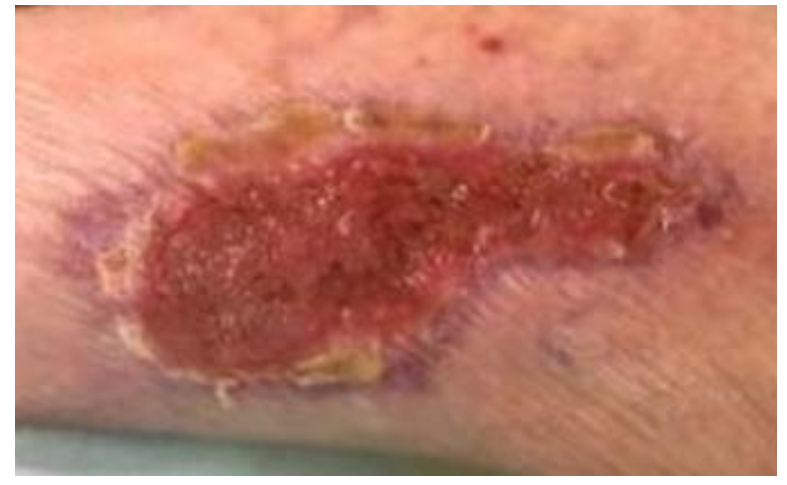

(b)

Figure 14. August 22, 2017, Week 9. (a) Pre-THOT ${ }^{\oplus}$. (b) Post-THOT ${ }^{\oplus}$. Wound measured $6 \times 2.5 \mathrm{~cm}$. Wound edges showed epithelial cells attached to the dermis and level with wound base. Epithelialization was observed at all edges. There was incomplete keratinization of the newly formed epithelial as shown by yellow scale at the wound edges. The differences in the yellow scale are probably due to cleaning of the wound, which causes the immature stratum corneum to lift off.

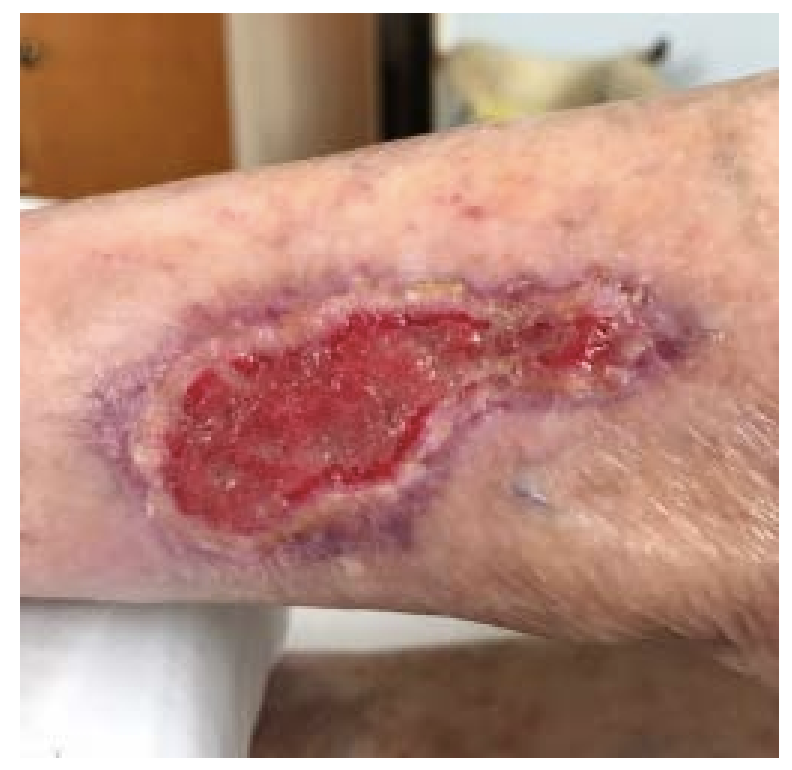

(a) 


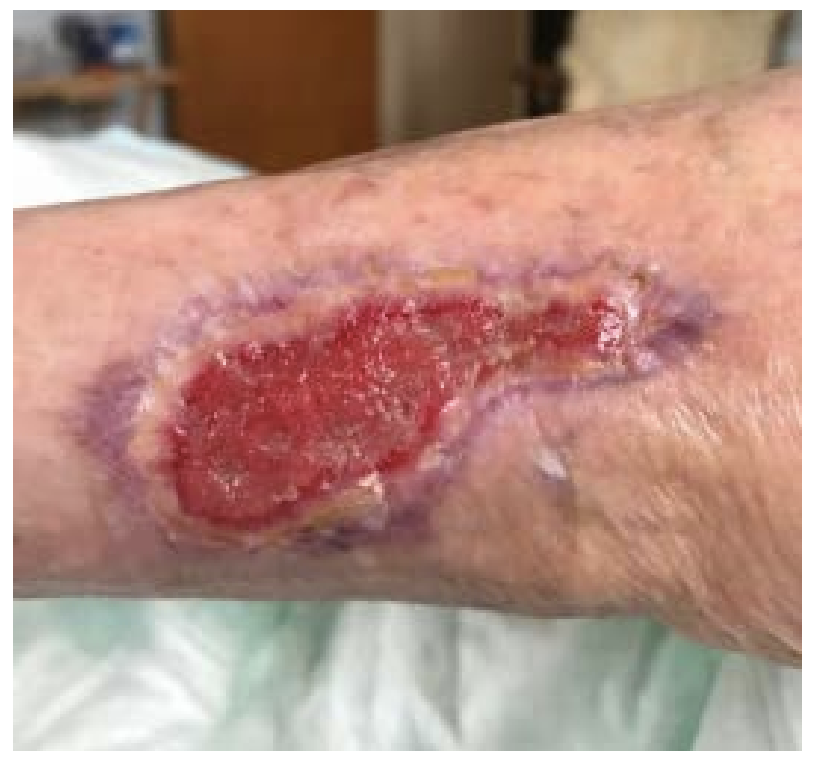

(b)

Figure 15. August 24, 2017, Week 9. (a) Pre-THOT ${ }^{\circledast}$ treatment. (b) Note well-defined wound base and well vascularized granulation tissue in the $\mathrm{THOT}^{\circledR}$-treated wound.

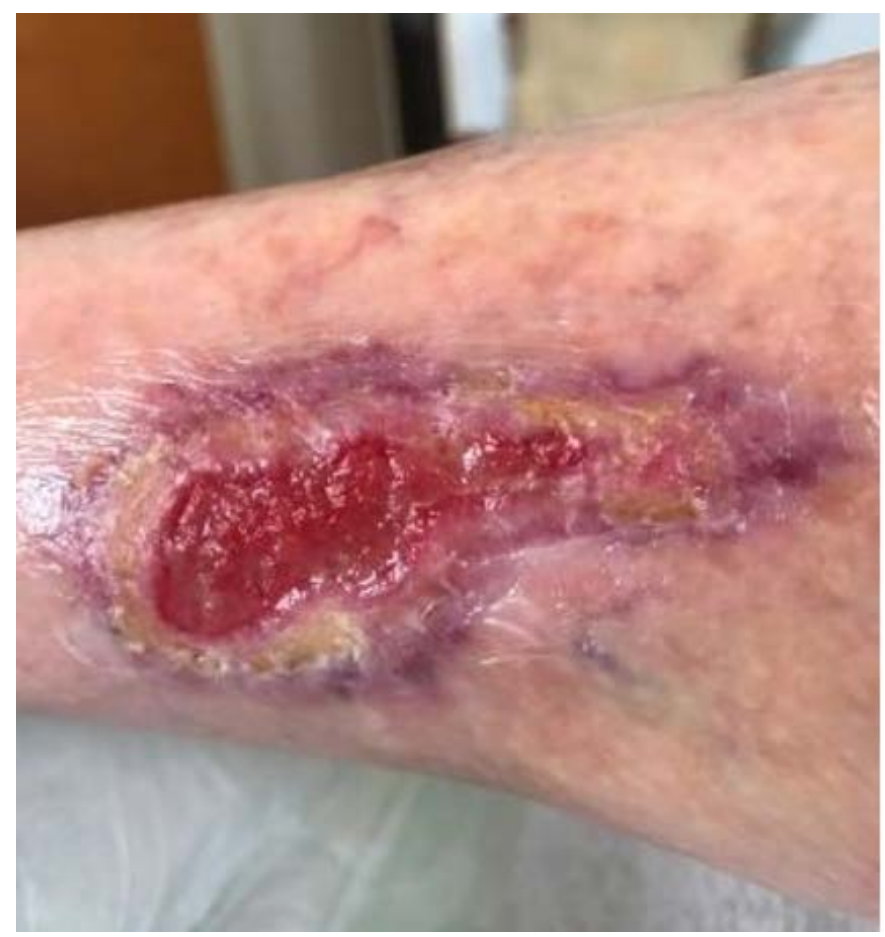

Figure 16. September 4, 2017, Week 10. Wound measured $3.5 \times 2 \mathrm{~cm}$. Wound edges were becoming indistinct in areas of re-epithelialization. Necrotic slough was no longer present. Wound exudate decreased significantly, with scant sanguineous drainage. Granulation tissue was observed to cover the entire base of the ulcer, with good epithelialization. There was no visible scarring i.e. formation of excess non-vascular fibrous tissue, suggesting the formation of good quality granulation tissue formed mainly of new blood vessels instead of abundant scar tissue with few blood vessels. Wounds healed with good quality granulation tissue tend not to break down again after discontinuation of THOT. 


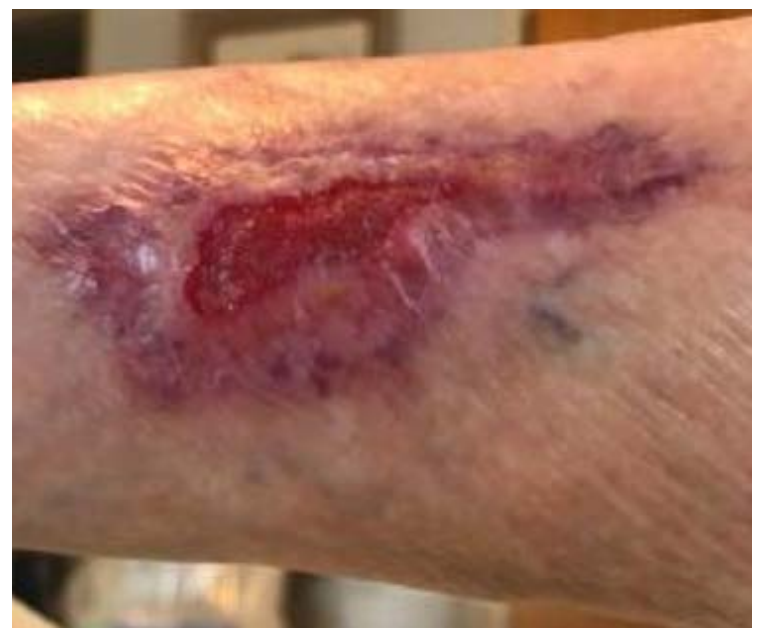

Figure 17. September 13, 2017, Week 12. Wound measured $3.5 \times 2 \mathrm{~cm}$. Wound appeared to be moist, without noticeable exudate.

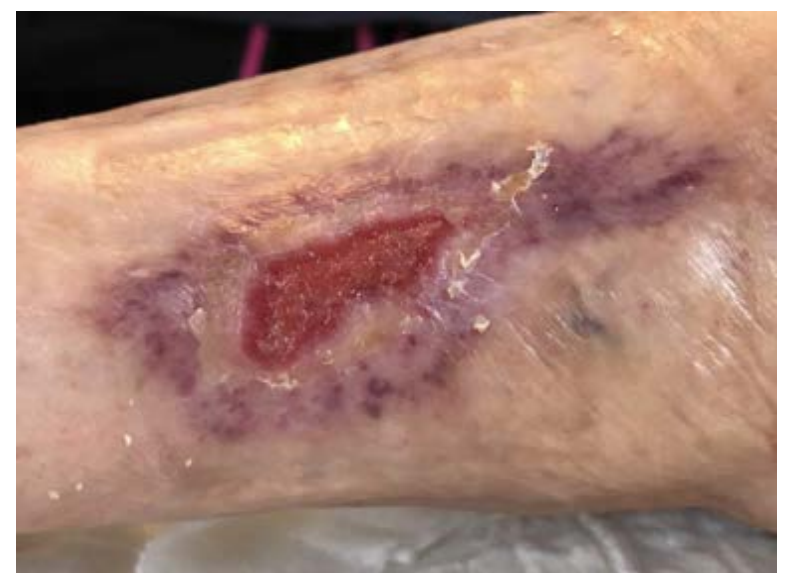

(a)

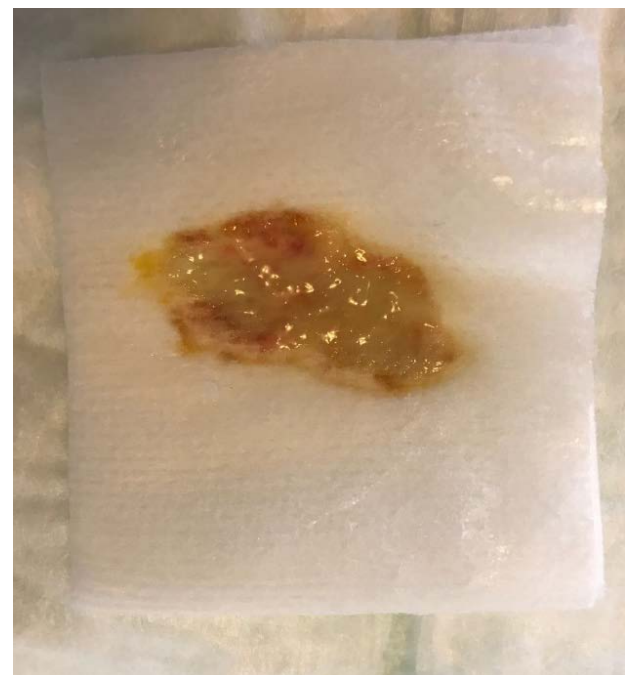

(b)

Figure 18. September 17, 2017, Week 12. (a) Epithelization was observed at the dermo-epithelial interphase. The ulcer measured $3 \times 1.8 \mathrm{~cm}$., with no leg edema or cellulitis. (b) Small amount of odorless sero-purulent exudate was observed on the dressings. 


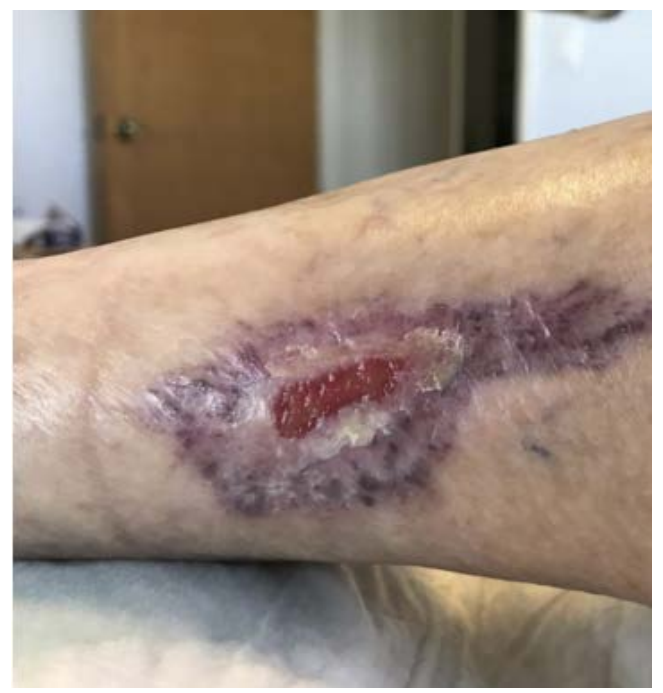

(a)

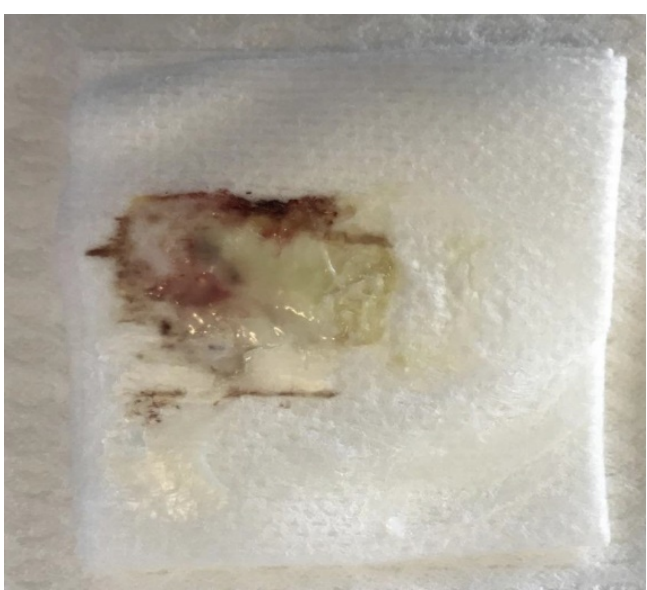

(b)

Figure 19. September 23, 2017, Week 13. (a) After 8 weeks of consecutive THOT $^{\circledast}$ treatment, THOT was paused for one week to reduce the risk of oxygen toxicity. The hyperpigmentation is probably due to both post-inflammatory hyperpigmentation as well as to hemosiderin from extravasated blood cells. The wound measured $2 \times 0.8 \mathrm{~cm}$. There was no evidence of local infection. (b) Scant odorless serosanguineous exudate was observed on the dressings.

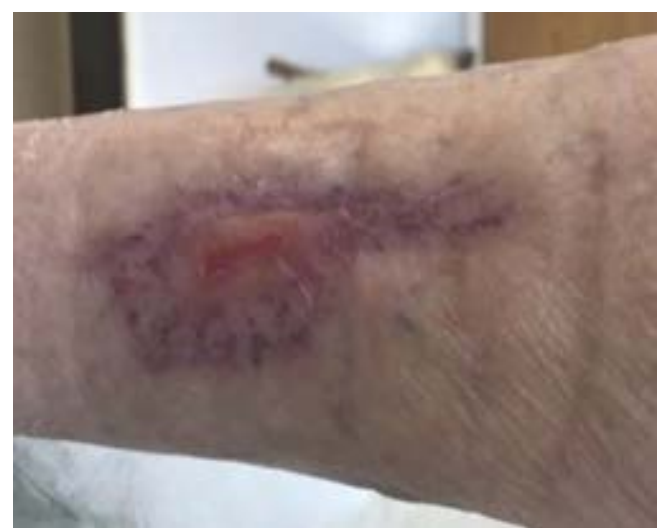

(a) 


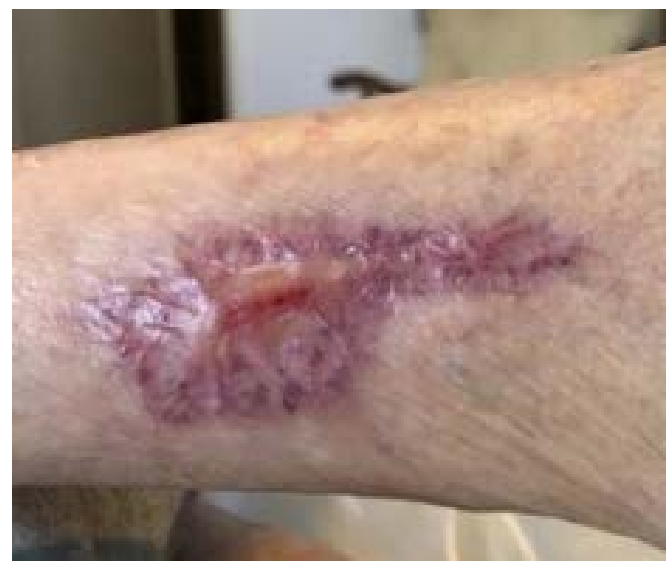

(b)

Figure 20. September 29, 2017, Week 14. (a) Pre-THOT ${ }^{\circledast}$ treatment after one-week THOT $^{\circledast}$ treatment break. (b) Post THOT $^{\circledast}$ treatment. Improved hydration, vascularization, and color was evident. The wound was completely healed with no dermis visible. The enlarged blood vessels were covered by partial thickness epidermis, accounting for the wrinkled appearance of the epidermis and increased visibility of the blood vessels. Carrasyn hydrogel dressings were added on September 27, 2017, to protect the wound, and prevent dehydration by maintaining a moist wound healing environment.

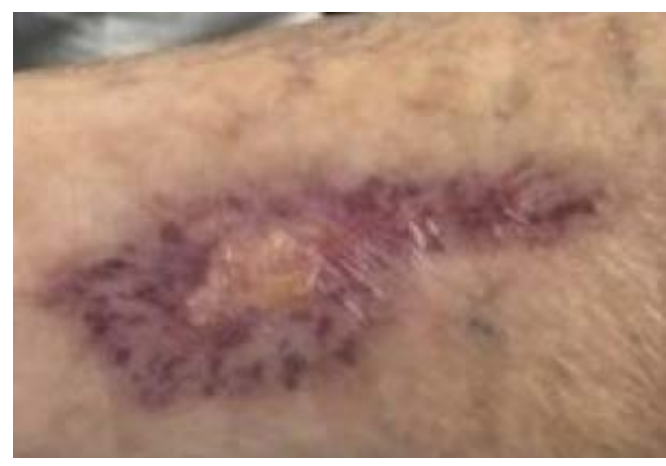

(a)

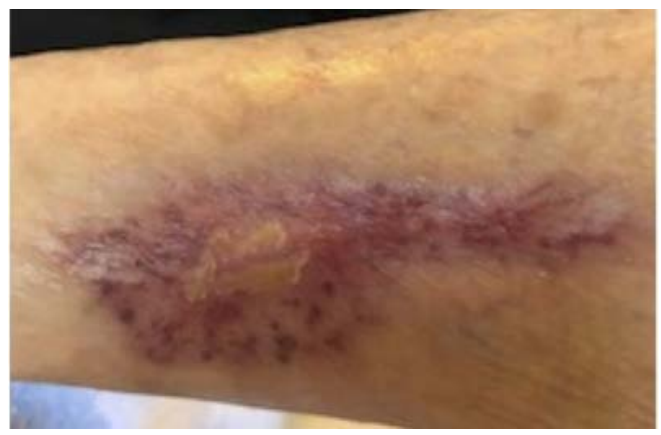

(b)

Figure 21. October 7, 2017, Week 15. (a) Pre-THOT ${ }^{\oplus}$ treatment. (b) Post THOT $^{\circledast}$ treatment. Wound fully closed, with epithelial tissue covering $100 \%$ of wound area. Sensation was intact. The epidermis was almost full thickness. Light tan thin yellow scale over the center of the wound denotes immature stratum corneum. After wound healing, the excess neovascularization diminishes and the capillaries decrease in size and number. This continues to take place over many months. 


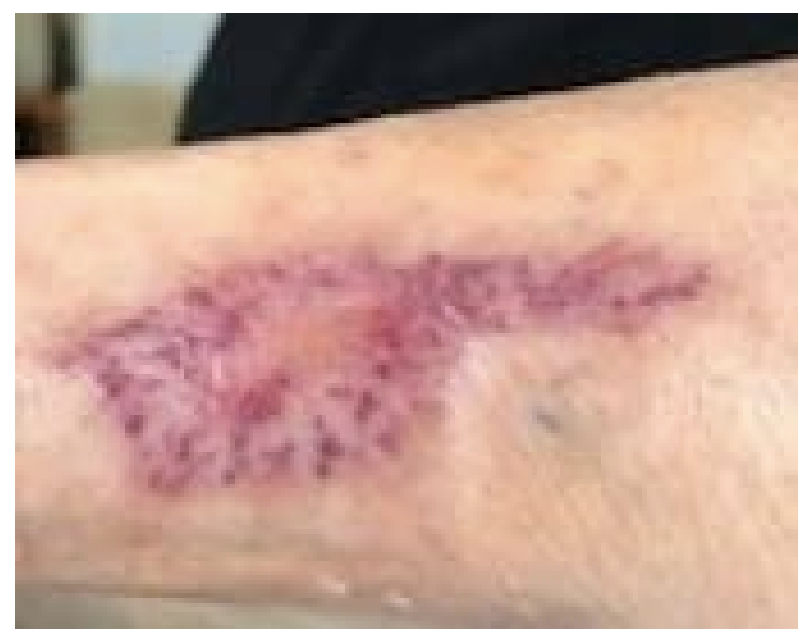

Figure 22. October 12, 2017, Week 16. Last THOT ${ }^{\oplus}$ treatment. Complete closure of wound after 15 weeks, with new epithelial tissue covering $100 \%$ of wound. Full sensation was intact, with no edema, cellulitis, scar tissue, or exudate. The patient was not in pain. Patient and caregivers were instructed to continue cleaning wound site daily with saline and to maintain gauze cover for protection as tissue remodeling and regeneration continued.

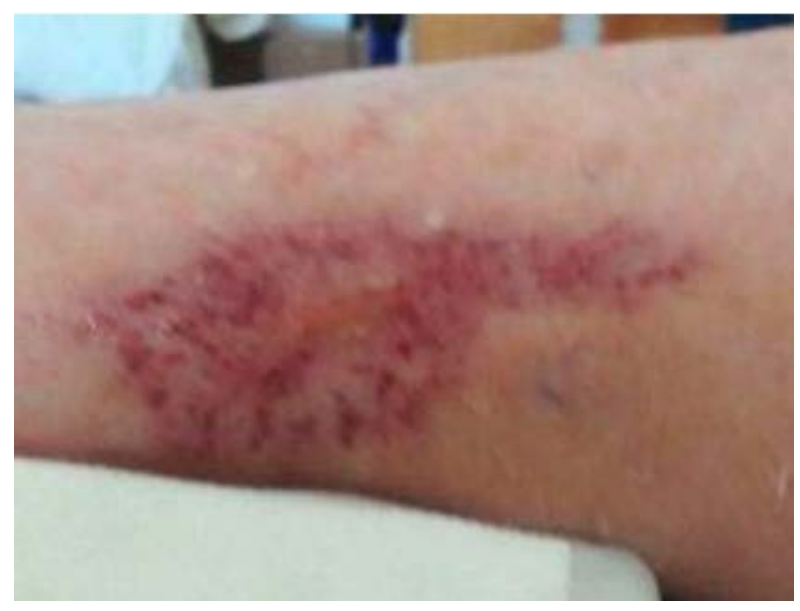

Figure 23. October 16, 2017. No sign of infection or skin breakdown. Good appearance and color.

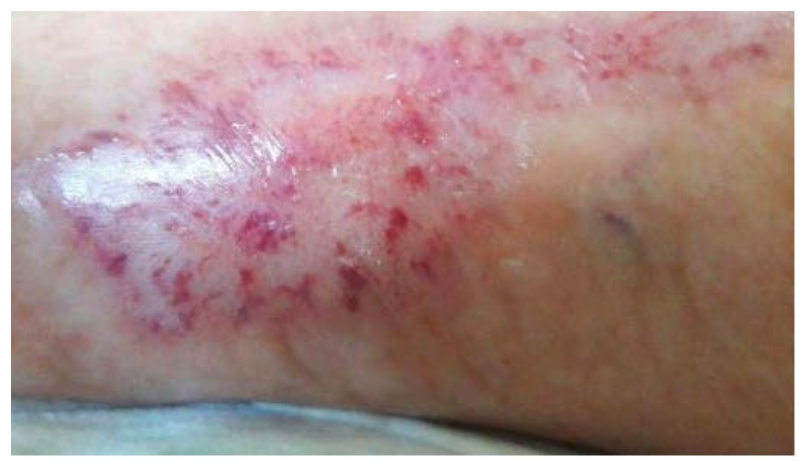

Figure 24. October 23, 2017. 11 days post $\mathrm{THOT}^{\circledR}-$ Skin condition and color indicate thickening of the epidermis overlying the wound with maturation of the stratum corneum (lack of scaling). 


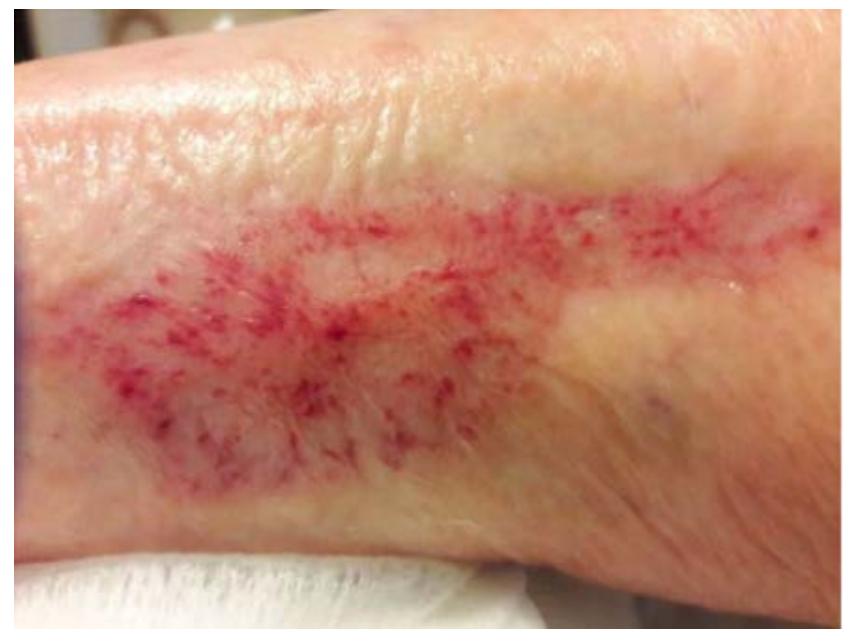

Figure 25. October 30, 2017, 3 weeks post THOT $^{\oplus}$ follow up.

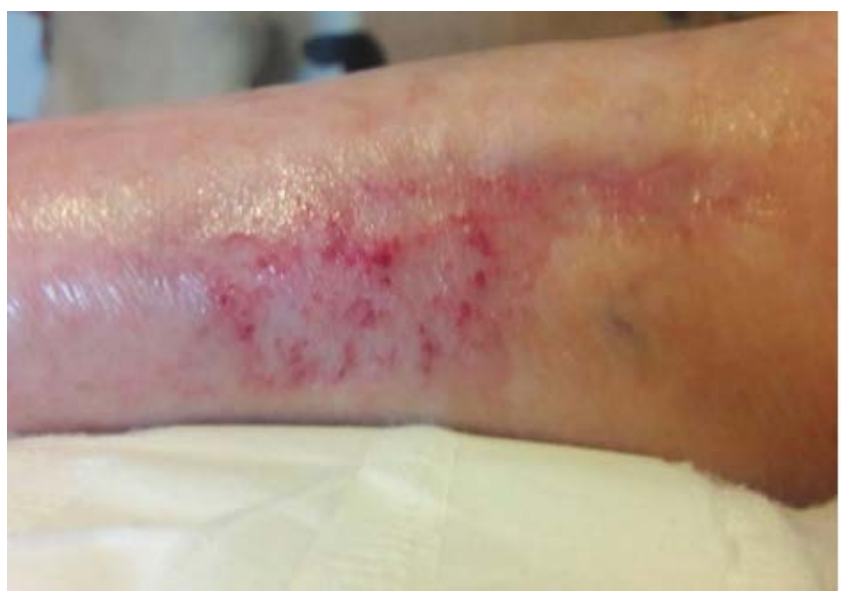

Figure 26. November 15, 2017. Week 5 post THOT ${ }^{\varpi}$. Note the presence of residual vascularization where the wound previously existed. The blood vessels were fewer, and less dilated than those observed previously since they were no longer needed for wound healing. The epidermis appeared to be approaching normal thickness, with mature, non-scaling stratum corneum. At this stage, the wound was considered to show complete regeneration of tissue and appearance of new skin.

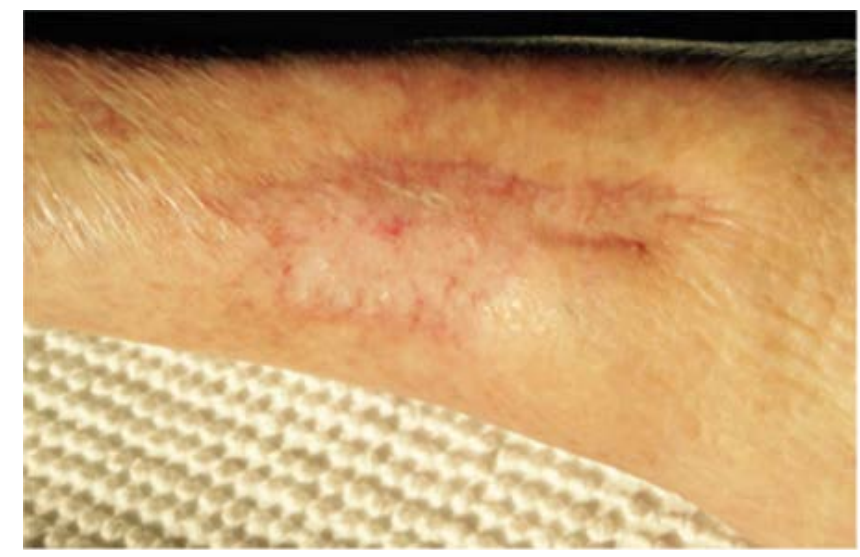

Figure 27. December 14, 2017. Week 9 post THOT $^{\oplus}$. Wound continued to exhibit the normal remodeling process after the wound had healed. 


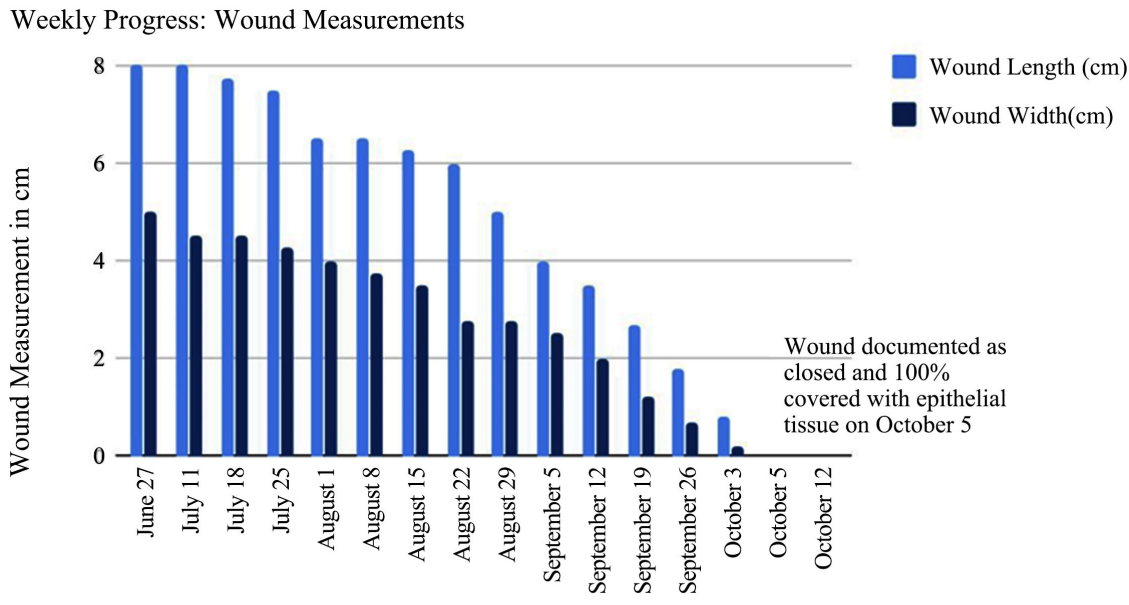

Date of Wound Assessment

Figure 28. Weekly wound measurements. The graph shows the reduction in wound size over time during treatment with $\mathrm{THOT}^{\varpi}$. In total, the patient received 15 weeks of topical hyperbaric oxygen therapy using the Numobag ${ }^{\oplus}$ Kit, and her stage IV chronic wound saw complete healing without the formation of scar tissue.

\subsection{Patient Follow-Up}

The patient was contacted for weekly follow-ups during the first two months after completing $\mathrm{THOT}^{\oplus}$, and again at six months post-treatment, reporting each time that the site of the preexisting wound remained healthy and fully healed.

\section{Discussion}

\subsection{Wound Healing with (THOT ${ }^{\circledR}$ )}

In this case study, the patient's wound was covered by a grey/black necrotic slough (Figure 1) suggesting a $\mathrm{TcPO}_{2}$ pressures at or below $13 \mathrm{~mm} \mathrm{Hg}$. The development of recurrent yellow necrotic slough after debridement (Figure 6 and Figure 7) supports the presence of tissue hypoxia with deficient blood supply. Angiogenesis induced by THOT $^{\circledR}$ (Figures 7-10), associated with decrease reformation of yellow necrotic slough, was also associated with re-epithelialization and decreased ulcer size (Figures 13-21). These findings are consistent with the notion that as the new blood vessels became more numerous, less necrotic tissue was formed from reperfusion injury, since free radical quenchers (superoxide dismutase, catalases and reduced glutathione) were contained within blood vessels, The turning point came when the number of new blood vessels provided free radical quenching properties that was sufficient to neutralize the free radicals produced by oxidative phosphorylation so that there was no further reperfusion injury when wound tissues were exposed to oxygen (Figures 13-21). At this point, no necrotic slough was formed, and the ATP generated by oxidative phosphorylation was used to stimulate further angiogenesis, collagen synthesis and re-epithelialization needed for wound healing.

It has been previously shown that in $\mathrm{THOT}^{\oplus}$ treated wounds, because there was abundant angiogenesis [18], there was relatively less scar tissue formed [18], 
since both tissues occupy the same space. This is an important feature since excessive scar tissue may compromise blood supply and lead to subsequent wound breakdown. In our patient, we observed that breakdown of the wound did not occur after THOT was discontinued. We believe that it was because the abundant new blood vessels generated by $\mathrm{THOT}^{\oplus}$ treatment was not associated with equally abundant formation of collagen fibers/scar tissue. The presence of good quality granulation tissue i.e. abundant angiogenesis (as measured by increased density of new blood vessels) without abundant scar tissue formation shown previously in THOT-treated biopsies [18], protects the wound from subsequent ischemia and wound break-down. Unlike wounds healed without THOT in which the granulation tissue contains abundant avascular scar tissue [18], the biopsies of granulation tissue in THOT-treated patients contained mainly endothelial cells and capillaries with sparse collagen fibers [18]. This ensures that once the wound is healed, there is no tendency for the wound to break down again.

The healing of the necrotic wound in our patient is primarily due to the adequacy of the vascularization induced by the $\mathrm{THOT}^{\oplus}$ treatment. This allows the vascularized granulation tissue to fill the space occupied by the necrotic/dead tissue in the dermis and deep fascia and allow for re-epithelialization of the healing wound. Even after the wound is healed, many changes continue to take place within the newly healed wound. 1) The new collagen fiber, which is secreted as single short fiber by dermal fibroblasts, lengthens and becomes thicker by cross-linking using S-S bonds. This makes the wound stronger and may take 3 months or more to occur. 2) Blood vessels and capillaries shrink in size and become fewer in number-they may take up to $1-1.5$ years to decrease in number. 3) The epidermis thickens, and the stratum corneum matures, and becomes less scaly. During this period remodeling of the tissues take place and inflammation decreases following wound repair (Figures 20-27).

\subsection{Review of the Literature}

Various methods of topical oxygen therapy exist, gaining traction over the past two decades. Some therapies deliver the oxygen at a normobaric pressure, while others, such as the one utilized in this case report, deliver the gas under a pressure slightly higher than atmospheric pressure and hence qualify as a "hyperbaric" oxygen treatment. In contrast to traditional hyperbaric oxygen therapy delivered in a chamber, topical hyperbaric oxygen therapy $\left(\mathrm{THOT}^{\circ}\right)$ is more portable, accessible, promotes patient comfort and quality of life, and carries significantly less risk of oxygen toxicity due to its lower pressure utilized and mode of delivery (topical rather than systemic). Pressures utilized by hyperbaric oxygen chambers are significantly higher (2 atmospheres) compared to $\mathrm{THOT}^{\oplus}$, with consequently higher risk of oxygen toxicity and reperfusion injury.

A review of the literature on topical hyperbaric oxygen therapy $\left(\mathrm{THOT}^{\oplus}\right)$ demonstrates convincing support for the efficacy of the therapy in healing wounds, 
as well as reveals the need for further clinical validation through well-designed randomized controlled studies. The effects of topical hyperbaric oxygen therapy were evaluated on stage II to IV ulcers in bed-ridden patients at a long-term care facility in one prospective randomized controlled clinical study (18). The study looked at 40 patients with 79 ulcers in total. The control group (50 ulcers) received standard wound care, which included pressure reduction techniques, antibiotic treatment, sharp debridement, and dressing changes as indicated. The treatment group (29 ulcers) received $\mathrm{THOT}^{\oplus}$ using a medical device and treatment regimen nearly identical to the one detailed in this case report. Results revealed healing in $90 \%$ of the ulcers receiving topical hyperbaric oxygen, while the standard wound care group saw only $22 \%$ achieve full healing. Tissue samples biopsied from the ulcers showed significantly increased capillary density in treatment group compared to the standard wound care group $(\mathrm{p}<0.001)$. Of note, 28 of the 29 ulcers in the THOT $^{\circledast}$ group, had no evidence of clinical scarring, in contrast to the observance of some degree of clinical scarring in 49 of the 50 ulcers in the standard wound care group. This study included a cost analysis which revealed impressive cost-savings for the $\mathrm{THOT}^{\oplus}$ group: at 4 weeks, stage II ulcers saw $81.3 \%$ savings compared to the standard wound care group, stage III ulcers saw $37.9 \%$ savings, and stage IV ulcers saw $36.1 \%$ savings [18].

The efficacy of $\mathrm{THOT}^{\oplus}$ on chronic diabetic foot ulcers was examined in a prospective, controlled study conducted at a single wound clinic [19]. Though not randomly assigned, the treatment and control groups were similarly comprised with regard to age, gender, $\mathrm{HbAlc}$ and $\mathrm{ABI}$. Baseline wound area for the treatment group was significantly greater $(\mathrm{p}=0.02)$, and average wound duration prior to treatment was longer (6.1 months [SD 5.8] versus 3.2 months [SD $0.4]$ for the control group), though not statistically significant. The treatment group $(n=17)$ received a form of topical hyperbaric oxygen therapy according to the device's protocol, for 60 minutes a day, 5 days a week. The control group $(\mathrm{n}=11)$ received advanced moist wound therapy with a Silvercel ${ }^{\mathrm{TM}}$ antimicrobial dressing, changed at the clinic twice weekly at minimum according to physician evaluation. All ulcers were debrided as clinically indicated and treated per best practice care guidelines. Results demonstrated that the treatment group was more likely to heal, with $82.4 \%$ of the treatment group achieving complete epithelialization compared to $45.5 \%$ of the control group ( $\mathrm{p}=0.04$ ). The study also found that the ulcers treated with topical hyperbaric oxygen therapy closed more quickly, as the median closure time was 56 days versus 93 days for the control group [19].

In a cohort study which compared $\mathrm{THOT}^{\oplus}$ to traditional hyperbaric oxygen therapy, 57 patients with chronic wounds were analyzed-32 receiving treatment in hyperbaric chambers, and 25 receiving topical oxygen therapy. Though enrollment was not randomized, the statistical approach to calculate treatment efficacy on wound closure was identical in both groups. Those treated with THOT $^{\oplus}$ saw significant wound closure $(\mathrm{p}=0.001)$, while those who received tra- 
ditional hyperbaric oxygen therapy did not demonstrate significant improvements in wound closure $(\mathrm{p}=0.150)$. Additionally, through analysis of tissue edge biopsies, topical oxygen therapy was associated with a higher VEGF expression [9].

\subsection{Cost Analysis of Wound Healing with THOT ${ }^{\circledR}$}

In addition to the Heng study referenced above, other studies have examined the cost savings of topical oxygen therapy. One case study series reported an average weekly cost of $\$ 700$ for the form of topical oxygen therapy utilized to treat pressure ulcers in patients with spinal cord injuries [20]. In comparison, the authors report a single session in the hyperbaric chamber ranges from $\$ 150$ to $\$ 1000$, and with chronic wounds often prescribed 5 to 7 sessions per week for treatment [21], the total weekly cost could reach $\$ 7000$ [20]. Further cost-effective studies are warranted comparing topical hyperbaric oxygen therapy to conventional wound treatments. However, when considering the evidence revealing topical oxygen therapy is less expensive than alternative treatment options and can lead to reduced healing time, this inevitably equates to a decrease in total treatment costs: less nursing care hours, fewer supplies required, reduced complications. Moreover, the portable nature of topical hyperbaric oxygen therapy allows for treatment in the home or clinic setting, reducing medical facility costs and patient transport expenses. Patients unable to receive traditional hyperbaric oxygen therapy due to mobility issues, lack of access to a hyperbaric chamber, or exclusion by contraindication could benefit greatly from the option of in-home topical hyperbaric oxygen therapy.

\subsection{Background of the Medical Device Used to Deliver THOT ${ }^{\circledR}$}

The Numobag ${ }^{\oplus}$ was developed through more than twenty years of clinical research and is FDA-approved. It is a thin, transparent, disposable membrane that covers the legs and approximately $75 \%$ of the human torso. Designed for single-use, each bag is composed of a polyethylene material that is considered "extremely clean", although not sterile. Tubing is used to connect the bag to an oxygen source. Once inflated fully, the Numobag ${ }^{\oplus}$ Kit delivers $100 \%$ medical-grade oxygen directly to the open wound at a pressure of 1.03 to 1.05 ATA, as according to the device's protocol.

\section{Summary and Conclusion}

Hypoxic wounds covered by black or yellow necrotic tissue are associated with insufficient blood supply leading to low $\mathrm{TcPO}_{2}$ values. Because of insufficient blood supply and the inability to neutralize oxygen free radicals, these wounds suffer reperfusion injury when exposed to oxygen in the air, with reformation of necrotic slough after debridement. These wounds are considered "non-healing" and usually require limb amputation. Our patient with a non-healing leg wound covered by black necrotic slough, with recurrent yellow slough formation fol- 
lowing debridement, was considered "non-healing" and scheduled for amputation. With THOT $^{\infty}$, the formation of new blood vessels with increased free radical quenching properties enabled the wound to heal because the tissues were no longer subjected to reperfusion injury.

The healing of the hypoxic non-healing wound with $\mathrm{THOT}^{\circledR}$ in our patient is an example that non-healing hypoxic wounds that may lead to limb amputations can be converted to non-hypoxic wounds capable of healing, with avoidance of amputation and increased limb salvage. In addition, the lack of excessive scar tissue in wounds healed with $\mathrm{THOT}^{\oplus}$ may prevent wound breakdown after healing.

On top of the successful results, THOT $^{\circledast}$ was a cost-effective option that promoted the quality of life of the patient and her support system. The treatment allowed the patient to remain in the comfort of her home and lessened the burden on caregivers who would have otherwise transported her to frequent wound care appointments. The medical device employed to deliver THOT $^{\circledast}$ in this case report has been used to effectively treat a variety of wounds, including burns, pressure injuries, diabetic ulcers, and necrotizing fasciitis, in the home, hospital, long-term care, and clinic setting. Today's chronic wound epidemic needs versatile therapies such as $\mathrm{THOT}^{\oplus}$, warranting further research and randomized controlled clinical studies to build the evidence base for this alternative treatment option. It must be emphasized that in our patient, without undergoing $\mathrm{THOT}^{\oplus}$, amputation would have proceeded as scheduled.

\section{Declarations}

\section{Acknowledgements}

The authors would like to thank the patient and her family for their enthusiasm and encouragement of the publication of this case report.

\section{Ethics Approval and Consent to Participate}

This case report was performed in accordance with international ethical rules.

\section{Consent for Publication}

Written informed consent was obtained from the patient for publication of this case report and any accompanying images. A copy of the written consent is available for review by the Editor-in-Chief of this journal.

\section{Funding}

Numotech, Inc. donated the Numobag ${ }^{\oplus}$ Kits during the complete course of treatment.

\section{Competing Interests}

The patient was referred for treatment with Hyperbaric Oxygen Therapy at Wound Care Center but was disqualified due to pre-existing conditions. 


\section{Availability of Data and Materials}

Data sharing is applicable to this article as datasets were generated or analyzed during the current study. Data support the results were extracted from the patient's medical records.

\section{Author's Contributions}

AH: management of the patient's $\mathrm{THOT}^{\varpi}$ clinical care team, review of the literature, data collection and analysis, and drafted the manuscript. SCB, RAD: served as part of the patient's clinical care team directly administering the topical hyperbaric oxygen therapy, data acquisition and analysis, revision of the manuscript. PMR: clinical consultant for the patient's THOT ${ }^{\oplus}$ clinical care team, data analysis, revision of the manuscript. VDL, MCYH Data analysis, manuscript preparation.

All authors read and approved the final manuscript.

\section{References}

[1] Frykberg, R.G. and Banks, J. (2015) Challenges in the Treatment of Chronic Wounds. Advances in Wound Care, 4, 560-582. https://doi.org/10.1089/wound.2015.0635

[2] Järbrink, K., Gao, N., Sönnergren, H., Schmidtchen, A., Pang, C., Bajpai, R., et al. (2017) The Humanistic and Economic Burden of Chronic Wounds: A Protocol for a Systematic Review. Systematic Reviews, 6, 15.

https://doi.org/10.1186/s13643-016-0400-8

[3] Sen, C.K., Gordillo, G.M., Roy, S., Kirsner, R., Lambert, L., Hunt, T.K., et al. (2009) Human Skin Wounds: A Major and Snowballing Threat to Public Health and the Economy. Wound Repair Regeneration, 17, 763-771.

https://doi.org/10.1111/j.1524-475X.2009.00543.x

[4] Nussbaum, S.R., Carter, M.J., Fife, C.E., DaVanzo, J., Haught, R., Nusgart, M., et al. (2018) An Economic Evaluation of the Impact, Cost, and Medicare Policy Implications of Chronic Non-Healing Wounds. Value in Health, 21, 27-32.

https://doi.org/10.1016/j.jval.2017.07.007

[5] Demling, R.H. (2009) Nutrition, Anabolism, and the Wound Healing Process: An Overview. Eplasty, 9, e9.

[6] Kimmel, H.M., Grant, A. and Ditata, J. (2016) The Presence of Oxygen in Wound Healing. Wounds, 28, 264-270.

[7] Hopf, H.W. and Rollins, M.D. (2007) Wounds: An Overview of the Role of Oxygen. Antioxidants and Redox Signaling, 9, 1183-1192.

https://doi.org/10.1089/ars.2007.1641

[8] Castilla, D.M., Liu, Z.J. and Velazquez, O.C. (2012) Oxygen: Implications for Wound Healing. Advances in Wound Care, 1, 225-230.

https://doi.org/10.1089/wound.2011.0319

[9] Gordillo, G.M., Roy, S., Khanna, S., Schlanger, R., Khandelwal, S., Phillips, G., et al. (2008) Topical Oxygen Therapy Induces VEGF Expression and Improves Closure of Clinically Presented Chronic Wounds. Clinical Experimental Pharmacology and Physiology, 35, 957-964. https://doi.org/10.1111/j.1440-1681.2008.04934.x

[10] Sen, C.K. (2009) Wound Healing Essentials: Let There Be Oxygen. Wound Repair 
Regeneration, 17, 1-18. https://doi.org/10.1111/j.1524-475X.2008.00436.x

[11] Bao, P., Kodra, A., Tomic-Canic, M., Golinko, M., Ehrlich, H.P. and Brem, H. (2009) The Role of Vascular Endothelial Growth Factor in Wound Healing. Journal Surgical Research, 153, 347-358. https://doi.org/10.1016/j.jss.2008.04.023

[12] Wounds UK (2017) Wounds UK Expert Panel Report. Consensus round Table Meeting. Clinical Pathway for Using Topical Oxygen Therapy in Practice.

http://www.wounds-uk.com/supplements/consensus-round-table-meeting-clinicalpathway-for-using-topical-oxygen-therapy-in-practice

[13] Rao, C., Xiao, L., Liu, H., Li, S., Lu, J., Li, J., et al. (2016) Effects of Topical Oxygen Therapy on Ischemic Wound Healing. Journal of Physical Therapy Science, 28, 118-123. https://doi.org/10.1589/jpts.28.118

[14] Levin, M.E. (1993) Pathogenesis and Management of Diabetic Foot Lesions. In: Levin, M.E., O’Neal, L.W. and Bowker, J.H., Eds., The Diabetic Foot, 5th Edition, Mosby, St Louis, 17-56.

[15] Heng, M.C.Y. (1993) Topical Hyperbaric Oxygen Therapy for Problem Wounds. Journal of Dermatology and Surgical Oncology, 19, 784-793.

https://doi.org/10.1111/j.1524-4725.1993.tb00425.x

[16] Jaesche, H., Smith, C.V. and Mitchell, J.R. (1988) Reactive Oxygen Species during Ichemia-Reflow Injury in Isolated Rat Liver. Journal of Clinical Investigation, 81, 1240-1246. https://doi.org/10.1172/JCI113441

[17] Zwier, J.L., Kauppusamy, P. and Lutry, G. (1988) Measurement of Endothelial Cell Free Radical Generation: Evidence for a Central Mechanism of Free Radical Injury in Post-Ischemic Tissue. Proceedings of the National Academy of Sciences, 85, 4046-4050. https://doi.org/10.1073/pnas.85.11.4046

[18] Heng, M.C.Y., Harker, J., Csathy, G., Marshall, C., Brazier, J., Sumampong, S., et al. (2000) Angiogenesis in Necrotic Ulcers Treated with Hyperbaric Oxygen. Ostomy Wound Management, 46, 18-32.

[19] Blackman, E., Moore, C., Hyatt, J., Railton, R. and Frye, C. (2010) Topical Wound Oxygen Therapy in the Treatment of Severe Diabetic Foot Ulcers: A Prospective Controlled Study. Ostomy Wound Management, 56, 24-31.

[20] Banks, P.G. and Ho, C.H. (2008) A Novel Topical Oxygen Treatment for Chronic and Difficult-to-Heal Wounds: Case Studies. Journal of Spinal Cord Medicine, 31, 297-301. https://doi.org/10.1080/10790268.2008.11760726

[21] Shah, J. (2010) Hyperbaric Oxygen Therapy. Journal of the American College of Certified Wound Specialists, 2, 9-13. https://doi.org/10.1016/j.jcws.2010.04.001

\section{Abbreviations}

USD: United States dollar

THOT $^{\circledast}$ : topical hyperbaric oxygen therapy

ATA: atmospheres absolute (standard atmosphere of pressure at sea level)

VEGF: vascular endothelial growth factor

ABI: ankle brachial index 